\title{
First constraint on coherent elastic neutrino-nucleus scattering in argon
}

D. Akimov, ${ }^{1,2}$ J. B. Albert, ${ }^{3}$ P. An,,${ }^{4,5}$ C. Awe,,${ }^{4,5}$ P. S. Barbeau,,${ }^{4,5}$ B. Becker, ${ }^{6}$ V. Belov,${ }^{1,2}$ M. A. Blackston, ${ }^{7}$ A. Bolozdynyaa ${ }^{2}$ B. Cabrera-Palmer, ${ }^{8}$ M. Cervantes, ${ }^{4}$ J. I. Collar, ${ }^{9}, 10$ R. L. Cooper, ${ }^{11,12}$ J. Daughhetee, ${ }^{6}$ M. del Valle Coello, ${ }^{3}$ J. A. Detwiler, ${ }^{13}$ M. D’Onofrio, ${ }^{3}$ Y. Efremenko, ${ }^{6,7}$ E. M. Erkela, ${ }^{13}$ S. R. Elliott, ${ }^{12}$ L. Fabris, ${ }^{7}$ M. Febbraro, ${ }^{7}$ W. Fox, ${ }^{3}$ A. Galindo-Uribarri, ${ }^{6,7}$ M. P. Green,${ }^{5,714}$ K. S. Hansen, ${ }^{13}$ M. R. Heath $\odot,{ }^{3, *}$ S. Hedges, ${ }^{4,5}$ T. Johnson, ${ }^{4,5}$ M. Kaemingk, ${ }^{11}$ L. J. Kaufman, ${ }^{3, \dagger}$ A. Khromov, ${ }^{2}$ A. Konovalov, ${ }^{1,2}$ E. Kozlova, ${ }^{1,2}$ A. Kumpan, ${ }^{2}$ L. Li,${ }^{4,5}$ J. T. Librande, ${ }^{13}$ J. M. Link ${ }^{15}$ J. Liu, ${ }^{16}$ K. Mann, ${ }^{5,7}$ D. M. Markoff, ${ }^{5,17}$ H. Moreno, ${ }^{11}$ P. E. Mueller, ${ }^{7}$ J. Newby, ${ }^{7}$ D. S. Parno, ${ }^{18}$ S. Penttila, ${ }^{7}$ D. Pershey, ${ }^{4}$ D. Radford, ${ }^{7}$ R. Rapp,${ }^{18}$ H. Ray, ${ }^{19}$ J. Raybern, ${ }^{4}$ O. Razuvaeva, ${ }^{1,2}$ D. Reyna, ${ }^{8}$ G. C. Rich, ${ }^{9}$ D. Rudik, ${ }^{1,2}$ J. Runge, ${ }^{4,5}$ D. J. Salvat, ${ }^{3,13}$ K. Scholberg, ${ }^{4}$ A. Shakirov, ${ }^{2}$ G. Simakov, ${ }^{1,2}$ G. Sinev, ${ }^{4}$ W. M. Snow, ${ }^{3}$ V. Sosnovtsev, ${ }^{2}$ B. Suh, ${ }^{3}$ R. Tayloe, ${ }^{3}$ K. Tellez-Giron-Flores, ${ }^{15}$

R. T. Thornton, ${ }^{3,12}$ I. Tolstukhin, ${ }^{3}$ J. Vanderwerp, ${ }^{3}$ R. L. Varner, ${ }^{7}$ C. J. Virtue ${ }^{20}$ G. Visser, ${ }^{3}$ C. Wiseman, ${ }^{13}$ T. Wongjirad, ${ }^{21}$ J. Yang, ${ }^{21}$ Y.-R. Yen, ${ }^{18}$ J. Yoo, ${ }^{22}$ C.-H. Yu, ${ }^{7}$ and J. Zettlemoyer ${ }^{3,7}$

${ }^{1}$ Institute for Theoretical and Experimental Physics named by A.I. Alikhanov of National Research Centre

"Kurchatov Institute", Moscow 117218, Russian Federation

${ }^{2}$ National Research Nuclear University MEPhI (Moscow Engineering Physics Institute), Moscow 115409, Russian Federation

${ }^{3}$ Department of Physics, Indiana University, Bloomington, Indiana 47405, USA

${ }^{4}$ Department of Physics, Duke University, Durham, North Carolina 27708, USA

${ }^{5}$ Triangle Universities Nuclear Laboratory, Durham, North Carolina 27708, USA

${ }^{6}$ Department of Physics and Astronomy, University of Tennessee, Knoxville, Tennessee 37996, USA

${ }^{7}$ Oak Ridge National Laboratory, Oak Ridge, Tennessee 37831, USA

${ }^{8}$ Sandia National Laboratories, Livermore, California 94550, USA

${ }^{9}$ Enrico Fermi Institute and Kavli Institute for Cosmological Physics, University of Chicago, Chicago, Illinois 60637, USA

${ }^{10}$ Department of Physics, University of Chicago, Chicago, Illinois 60637, USA

${ }^{11}$ Department of Physics, New Mexico State University, Las Cruces, New Mexico 88003, USA

${ }^{12}$ Los Alamos National Laboratory, Los Alamos, New Mexico 87545, USA

${ }^{13}$ Center for Experimental Nuclear Physics and Astrophysics \& Department of Physics, University of Washington, Seattle, Washington 98195, USA

${ }^{14}$ Department of Physics, North Carolina State University, Raleigh, North Carolina 27695, USA

${ }^{15}$ Center for Neutrino Physics, Virginia Tech, Blacksburg, Virginia 24061, USA

${ }^{16}$ Physics Department, University of South Dakota, Vermillion, South Dakota 57069, USA

${ }^{17}$ Department of Mathematics and Physics, North Carolina Central University, Durham, North Carolina 27707, USA

${ }^{18}$ Department of Physics, Carnegie Mellon University, Pittsburgh, Pennsylvania 15213, USA

${ }^{19}$ Department of Physics, University of Florida, Gainesville, Florida 32611, USA

${ }^{20}$ Department of Physics, Laurentian University, Sudbury, Ontario P3E 2C6, Canada

${ }^{21}$ Department of Physics and Astronomy, Tufts University, Medford, Massachusetts 02155, USA

${ }^{22}$ Department of Physics at Korea Advanced Institute of Science and Technology (KAIST)

and Center for Axion and Precision Physics Research (CAPP) at Institute for Basic Science (IBS),

Daejeon 34141, Republic of Korea

(Received 19 September 2019; published 9 December 2019)

Coherent elastic neutrino-nucleus scattering (CEvNS) is calculated to be the dominant neutrino scattering channel for neutrinos of energy $E_{\nu}<100 \mathrm{MeV}$. We report a limit for this process from data collected in an engineering run of the $29 \mathrm{~kg}$ CENNS-10 liquid argon detector located $27.5 \mathrm{~m}$ from the pion decay-at-rest neutrino source at the Oak Ridge National Laboratory Spallation Neutron Source (SNS) with $4.2 \times 10^{22}$ protons on target. The dataset provided constraints on beam-related backgrounds critical for future measurements and yielded $<7.4$ candidate CEvNS events which implies a cross section for the

*heathmr@ornl.gov

${ }^{\dagger}$ Present address: SLAC National Accelerator Laboratory, Menlo Park, California 94205, USA.

Published by the American Physical Society under the terms of the Creative Commons Attribution 4.0 International license. Further distribution of this work must maintain attribution to the author(s) and the published article's title, journal citation, and DOI. Funded by $S C O A P^{3}$. 
process, averaged over the SNS pion decay-at-rest flux, of $<3.4 \times 10^{-39} \mathrm{~cm}^{2}$, a limit within twice the Standard Model prediction. This is the first limit on CEvNS from an argon nucleus and confirms the earlier $\mathrm{CsI}[\mathrm{Na}]$ nonstandard neutrino interaction constraints from the collaboration. This run demonstrated the feasibility of the ongoing experimental effort to detect CEvNS with liquid argon.

DOI: 10.1103/PhysRevD.100.115020

\section{INTRODUCTION}

Coherent elastic neutrino-nucleus scattering (CEvNS), predicted in 1974 as a consequence of the neutral weak current $[1,2]$, is calculated to be the dominant neutrino interaction for neutrinos of energy $E_{\nu}<100 \mathrm{MeV}$. The cross section has a characteristic dependence on the square of the number of neutrons $\left(N^{2}\right)$ reflecting the coherent sum of the weak charge carried by the neutrons and is sensitive to nuclear physics effects [3-8] through the nuclear form factor, $\left[F\left(Q^{2}\right)\right]$, as seen in the differential cross section for a spin-zero nucleus [3]:

$\frac{d \sigma}{d T}=\frac{G_{F}^{2} M}{2 \pi}\left[2-\frac{2 T}{E_{\nu}}+\left(\frac{T}{E_{\nu}}\right)^{2}-\frac{M T}{E_{\nu}^{2}}\right] \frac{Q_{W}^{2}}{4} F^{2}\left(Q^{2}\right)$

where $T$ is the recoil energy, $M$ is the mass of the nucleus, and $Q_{W}=N-Z\left(1-4 \sin ^{2} \theta_{W}\right)$ is the weak charge with weak mixing angle $\theta_{W}$. CEvNS is also sensitive to physics beyond the Standard Model (SM) [9-14]. In particular, the ability of a CEvNS measurement to constrain so-called "nonstandard interactions" (NSI) is critical as their presence can confound the mass ordering determination by longbaseline neutrino experiments such as DUNE [15-17].

CEvNS has eluded detection until recently because of the challenging technical requirements: $\mathcal{O}(10 \mathrm{keV})$ nuclear recoil energy thresholds, intense sources/large target masses, and low backgrounds. The COHERENT collaboration has recently overcome these challenges with stateof-the-art detector technology combined with the intense, pulsed, stopped-pion neutrino source available at the Spallation Neutron Source (SNS) at Oak Ridge National Laboratory (ORNL), using a CsI[Na] crystal to achieve the first measurement of CEvNS [18].

The next step for this program is a demonstration of the $N^{2}$ cross section dependence via observation of the process in other nuclei. To that end, the $29 \mathrm{~kg}$ liquid argon detector CENNS-10 was commissioned as part of the COHERENT experiment. We report here results from CENNS-10 as configured for an initial engineering run to establish the scintillation response, light yield, and energy calibration of the detector, as well as characterize the expected backgrounds. The results reported here provided the first experimental limit on the CEvNS cross section with argon nuclei, and they informed a detector upgrade for a longerterm CEvNS search with improved light yield and background reduction.

\section{EXPERIMENT}

The ORNL SNS produces neutrons via a $1.4 \mathrm{MW}$, roughly $1 \mathrm{GeV}$ proton beam pulsed at $60 \mathrm{~Hz}$ on a liquid-Hg target (with a typical proton beam trace having a $\mathrm{FWHM}=360 \mathrm{~ns}$ ). This beam also produces copious charged pions leading to a large neutrino flux via $\pi^{+}$decayat-rest (DAR). Although the total integrated beam power may be known to within $1 \%$, the total neutrino flux is only known to $10 \%$ because of systematic uncertainties in the pion production rate at the SNS, predicted to be $0.09 \pi^{+}$ for each proton-on-target (POT) at the beam energy for this run period [18]. These $\pi^{+}$produce a prompt $(\tau=26 \mathrm{~ns})$ $29.8 \mathrm{MeV} \nu_{\mu}$ along with a $\mu^{+}$which then decays, yielding a delayed $(\tau=2.2 \mu \mathrm{s}) 3$-body spectrum of $\bar{\nu}_{\mu}, \nu_{e}$, and $e^{+}$ with an endpoint of $53 \mathrm{MeV}$. The $\pi^{-}$production rate is roughly half the $\pi^{+}$rate. Nearly $99 \%$ of the $\pi^{-}$produced stop and are captured in the half meter thick high- $Z$ target yielding a very pure $\pi^{+}$DAR neutrino flux. The pulsed nature of the SNS beam allows for a large reduction in beam-unrelated backgrounds for neutrino experiments.

After a campaign of background measurements in the SNS experimental hall, a low-background area in a basement corridor was identified as a suitable area in which to measure CEvNS. This corridor ("neutrino alley"), is shielded by $\gtrsim 20 \mathrm{~m}$ of concrete and gravel from the SNS target assembly and by 8 meter water equivalent overburden. This provides a space with a low total background rate and, in particular, a sufficiently low beam-relatedneutron rate for a measurement of CEvNS.

In late 2016 the CENNS-10 detector, a single-phase liquid-argon (LAr) scintillation detector (Fig. 1) [19], was installed in neutrino alley $27.5 \mathrm{~m}$ from the SNS target. CENNS-10 was initially built at Fermilab for a prototype experiment [20] to run near the Fermilab Booster neutrino source. It contains a total LAr mass of $79.5 \mathrm{~kg}$.

For this engineering run, a $29 \mathrm{~kg}$ active detector mass was defined by a surrounding acrylic cylindrical shell coated with $0.2 \mathrm{mg} \mathrm{cm}^{-2} \mathrm{TPB}$ (tetraphenyl-butadiene) to wavelength-shift the $128 \mathrm{~nm}$ argon scintillation light to a distribution with $\lambda_{\text {peak }} \approx 400 \mathrm{~nm}$ [21-24]. This visible light was viewed with two 8" diameter Hamamatsu R591202MOD photomultiplier tubes (PMTs) read out with a CAEN V1720 $250 \mathrm{MHz}$ digitizer. The LAr, cooled and liquified with a $90 \mathrm{~W}$ Cryomech PT-90 cold head, was contained in a stainless-steel detector vessel within a vacuum cryostat. As seen in Fig. 1, the cryostat was suspended in a 


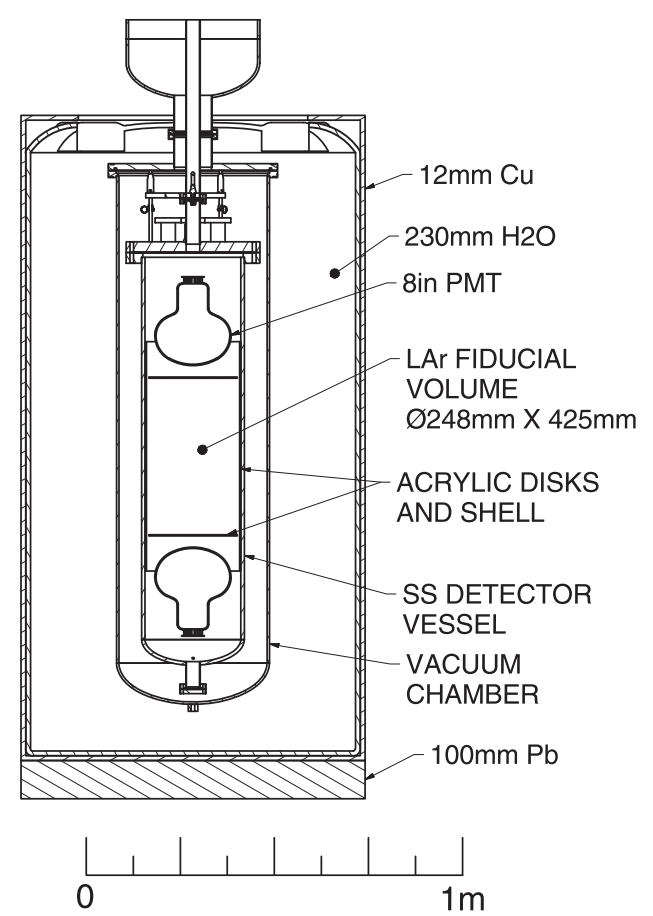

FIG. 1. CENNS-10 liquid argon detector and shielding as configured for this engineering run.

cylindrical water tank which was further contained within an external copper layer sitting on a layer of lead. The water layer reduces the beam-related neutron backgrounds, the lead is designed to reduce the flux from environmental $\gamma$ backgrounds, and the copper is added to shield from x-rays produced from ${ }^{210} \mathrm{~Pb} \beta$ decays in the lead.

This engineering run coincided with 3 months of SNS neutron production corresponding to a total integrated beam power of $1.8 \mathrm{GWh}\left(4.2 \times 10^{22} \mathrm{POT}\right)$ at an average energy of $973 \mathrm{MeV}$. A CEvNS search was performed with $1.5 \mathrm{GWh}$ of beam following the completion of the fullshielding (water and copper) installation. Data were read from the digitizer in $33 \mu \mathrm{s}$ windows centered around the $60 \mathrm{~Hz}$ beam spills. In addition to these "beam" triggers, identical windows ("strobe" triggers) were read asynchronously with the beam spills to precisely characterize beamunrelated events.

LAr is a natural choice as a medium to detect CEvNS. It provides a light nucleus in contrast to CsI to test the $N^{2}$ dependence of the CEvNS cross section. Argon has been widely used for both dark matter WIMP searches $[25,26]$ and for neutrino detection [27] and has therefore been well characterized above $20 \mathrm{keV}$ in the literature. It has a high light yield, 40 photons/keVee [28] (electron equivalent energy deposition), providing a sufficiently low threshold for CEvNS detection, and the quenched response to nuclear recoils has been well-characterized [29-32] allowing for well-understood CEvNS predictions. LAr scintillates on two significantly different timescales $\left(\tau_{\text {singlet }} \approx 6 \mathrm{~ns}, \tau_{\text {triplet }} \approx\right.$ $1600 \mathrm{~ns})$ [33] providing powerful pulse-shape discrimination

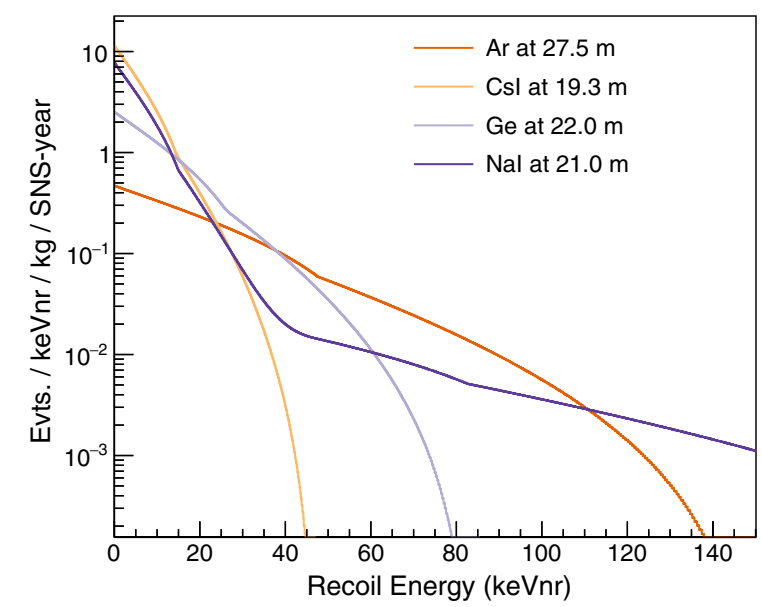

FIG. 2. Nuclear recoil kinetic energy distribution from CEvNS for the SNS pion decay-at-rest neutrino spectrum for currently deployed and planned COHERENT detectors at their respective detector locations in Neutrino Alley. The change in slope at roughly $50 \mathrm{keV}$ in the argon curve is due to the endpoint of the recoils caused by the prompt $\nu_{\mu}$ flux.

(PSD) capabilities to separate nuclear from electronic recoils (NR and ER respectively) [34-36]. Both the light output and PSD capabilities depend on the LAr purity.

As seen in Fig. 2, the CEvNS process in LAr with the SNS neutrino source produces nuclear recoils up to $\sim 100 \mathrm{keVnr}$ (nuclear recoil). Owing to the low-energy recoil signal and low event rates, the expected backgrounds need to be well characterized. In neutrino alley, CENNS-10 is sensitive to both beam-related and beam-unrelated backgrounds. These beam-unrelated backgrounds typically cause electronic recoils and are dominated by a high flux of $511 \mathrm{keV}$ gamma rays from a pipe running through neutrino alley carrying radioactive gas from the SNS target system. The PSD capabilities of LAr are used to reject most of these events; the rate of those remaining in the sample is measured via the strobe windows. In a strict sense, these $511 \mathrm{keV}$ gamma rays are beam-related and their rates change with the time history of accelerator operations. However, because the rate of change is small compared to the beam pulse rate, they are characterized as beam unrelated. External beam-unrelated backgrounds have largely been mitigated in a subsequent run of CENNS-10 with the installation of additional $\mathrm{Pb}$ shielding, making ${ }^{39} \mathrm{Ar}$ the dominant beam-unrelated background. The ${ }^{39} \mathrm{Ar}$ isotope is cosmogenically produced and is inherent in atmospheric sources of Ar. COHERENT is considering the use of underground argon depleted in ${ }^{39} \mathrm{Ar}$ [37-39] for future LAr measurements.

A more challenging background for a CEvNS analysis is caused by beam-related neutrons produced in the SNS target. Beam-related neutrons arrive in-time with the SNS beam pulse and elastically scatter off argon nuclei, generating nuclear recoils and mimicking the CEvNS signal. To characterize the beam-related neutron flux in energy 
and time, it was measured by the SciBath detector $[20,40]$ at the CENNS-10 location in late 2015. This measurement indicated that the beam-related neutron flux in time with the beam pulse is substantial compared to the prompt CEvNS signal, whereas the delayed beam-related neutron flux is negligible, providing a suitable time window to search for CEvNS [41].

\section{ANALYSIS}

The analysis of this dataset proceeded as follows: First a suite of radioactive gamma and neutron sources were used to calibrate the detector energy and PSD response and the detector simulation was tuned to match these data. Then beam-unrelated backgrounds were measured with strobe triggers, the beam-related background from beam-related neutrons was predicted with simulation based on the previous SciBath measurement, and the CEvNS signal was predicted from the SM cross section. Energy, PSD, and time cuts were then optimized with those estimates to maximize beam-related signal significance. With those cuts, a reduced neutron-shielding dataset was used to adjust the beam-related neutron prediction for the full shielded configuration. Finally, cuts were optimized and fixed for both a "counting experiment" and a likelihood fit before analyzing the full shielded beam-on dataset. While the light output was not sufficient for a sensitive CEvNS search in this initial engineering run, event excesses in a higher energy window are reported to characterize the beamrelated neutrons. In addition, the first limit on the CEvNS cross section in argon is placed and implications for NSI investigated.

Each individual digitized PMT waveform was analyzed for every trigger in the data stream and saturated waveforms were removed from the dataset. A baseline was determined from the average analog-to-digital converter (ADC) value in the first $1 \mu \mathrm{s}$ of each remaining waveform. This baseline is then used to identify PMT pulses on each channel above a 20 ADC count ( $\sim 2$ photoelectron) threshold. Events are identified when there are coincident PMT signals above this threshold to avoid triggering on single photoelectronlevel pulses from PMT dark rate. A requirement that the maximum ADC value occur within the first $90 \mathrm{~ns}$ of the event minimizes the effects from event pileup. A local baseline is calculated immediately before each pulse and a least-squares parabola fit is performed to the pulse peak for an accurate singlet pulse-height measurement. The results from the parabola fit are used to fit a single photoelecton template shape to the singlet peak and the residual between the single photoelectron template and the data is taken. Finally, the integral of the residual waveform is taken as a measure of the amount of triplet light in the event. A pulse shape parameter ( $F_{\text {prompt }}$ defined as the ratio of singlet to total light) can then be calculated to separate ER background events from the NR CEvNS signal.
Weekly calibration datasets with a ${ }^{137} \mathrm{Cs}$ source were used to measure the detector light output as well as track any changes during the course of this run. The detected photon yield was $0.6 \mathrm{PE} / \mathrm{keVee}$ as determined from the observed $662 \mathrm{keV}$ photopeak from the summed singlet and triplet light in the ${ }^{137} \mathrm{Cs}$ spectrum (Fig. 3). With the use of the ${ }^{137} \mathrm{Cs}$ datasets, the triplet lifetime in CENNS-10 was measured to be $\mathcal{O}(1.2 \mu \mathrm{s})$, consistent with an impurity level on the order of $\mathcal{O}(1 \mathrm{ppm})$ [42], adequate for a scintillationonly detector. It should be noted that the light yield was increased by a factor of 8 in a subsequent upgrade of this detector.

Monthly datasets collected with a ${ }^{252} \mathrm{Cf}$ fission source were used to characterize the response of CENNS-10 to NR events. The separation of NR and ER events in the ${ }^{252} \mathrm{Cf}$ dataset is shown in Fig. 4, where the band at low $F_{\text {prompt }}$ is identified as due to ER events and that at high $F_{\text {prompt }}$ is identified as NR events due to the fission neutrons. The observed $F_{\text {prompt }}$ is consistent with the expected singlet: triplet ratios of ER and NR events [33].

These calibration datasets enabled the tuning of the CENNS-10 Geant4-based [43] Monte Carlo (MC) simulation optical properties for both ER and NR events. These detector simulations were used to evaluate the efficiency for low-energy NR events to be detected and to form predictions of the expected beam-related neutron and CEvNS event rates in CENNS-10. An energy-independent fit over the energy range of interest to the global LAr data on nuclear recoil scintillation quenching [29-32] provided a quenching factor $(0.289 \pm 0.035)$ for NR vs ER response in CENNS-10. With these waveform analysis and calibration procedures, each detector event can be identified as an ER or NR candidate and be assigned a corresponding energy with units of keVee or keVnr.

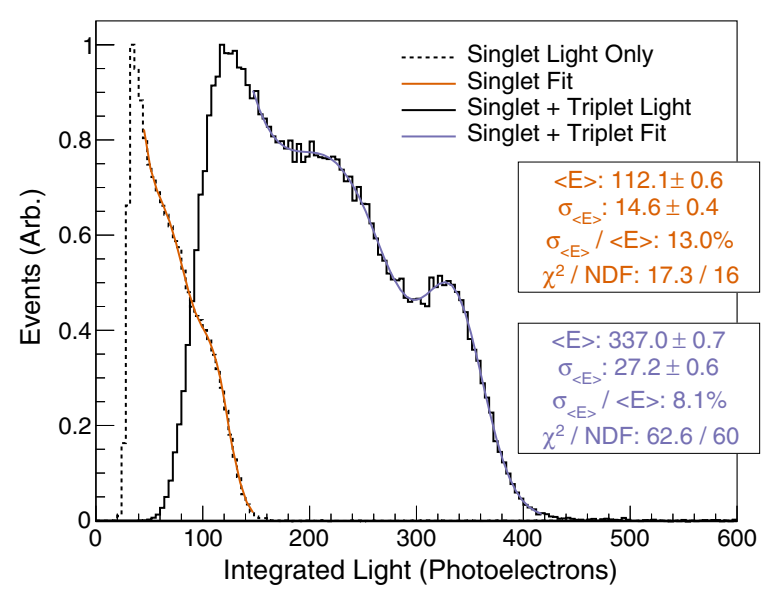

FIG. 3. Reconstructed energy spectrum with a ${ }^{137} \mathrm{Cs}$ calibration source. The $662 \mathrm{keV}$ photopeak is used to characterize the detected photon yield of $0.6 \mathrm{PE} / \mathrm{keVee}$ for this initial engineering run. The singlet pulse fitting allows for the singlet light to be summed separately. 


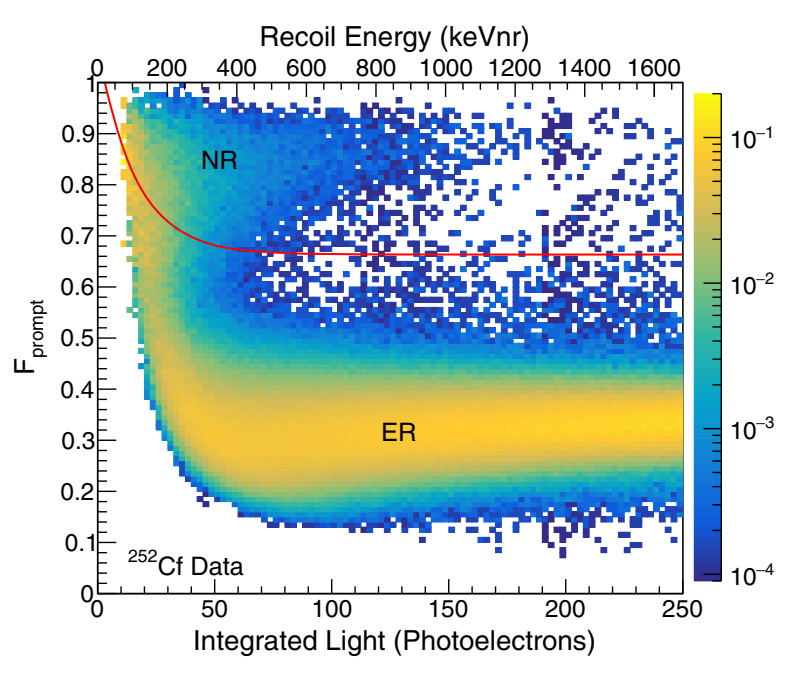

FIG. 4. Distribution of the $F_{\text {prompt }}$ parameter as a function of detected light in ${ }^{252} \mathrm{Cf}$ calibration data with decays to both neutrons and gammas. The overlaid red curve is a PSD cut optimized for the cuts-based counting experiment analysis discussed in the text.

Initial beam-related neutron predictions using a simulation based on the 2015 SciBath measurement were compared to a dedicated 2-week minimal neutron-shielding dataset. From this comparison, the predicted beam-related neutron rate was found to be $20 \%$ lower than the observed rate. This factor was used to adjust the expected neutron rates for the primary CEvNS dataset. However, the beamrelated neutron normalization was allowed to float in the final analysis. CEvNS predictions were based on the convolution of the pion DAR neutrino flux and SNS pion-production rate [18] with the SM-predicted CEvNS cross section. Beam-unrelated backgrounds were measured in situ with strobe triggers.

Both a cuts-based ("counting experiment") analysis and a likelihood fit in energy, time, and $F_{\text {prompt }}$ space were performed on the full-shielded CEvNS dataset. In the cutsbased analysis, to form a CEvNS sample, a figure-of-merit $\mathcal{F} \equiv N_{\text {sig }} / \sigma_{\text {sig }}$ was optimized to set a $0-30$ keVee reconstructed energy range, a delayed $1.4<t_{\text {Trig }}<4.4 \mu$ s time window (where $t_{\text {Trig }}$ is measured relative to a timing signal provided by the SNS close to the onset of POT), and an energy-dependent PSD selection seen in Fig. 4. For this analysis, it was assumed that the beam-related neutrons observed in neutrino alley are produced by fast neutrons from the target scattering in the shielding near the detector and that the neutrinos should arrive roughly $30 \mathrm{~ns}$ before the fast neutron peak determined from the beam-related neutron measurements. The results reported here are not sensitive to this assumption. A beam-related-neutronenhanced sample was also selected with an expanded energy range $(0-700 \mathrm{keVee})$ in both the prompt $\left(0.4<t_{\text {Trig }}<\right.$ $1.4 \mu \mathrm{s})$ and the delayed $\left(1.4<t_{\text {Trig }}<4.4 \mu \mathrm{s}\right)$ time windows.

For the likelihood fit, cuts were loosened, increasing the sensitivity to a CEvNS signal, to 0-300 keVee, 0.4-4.4 $\mu \mathrm{s}$

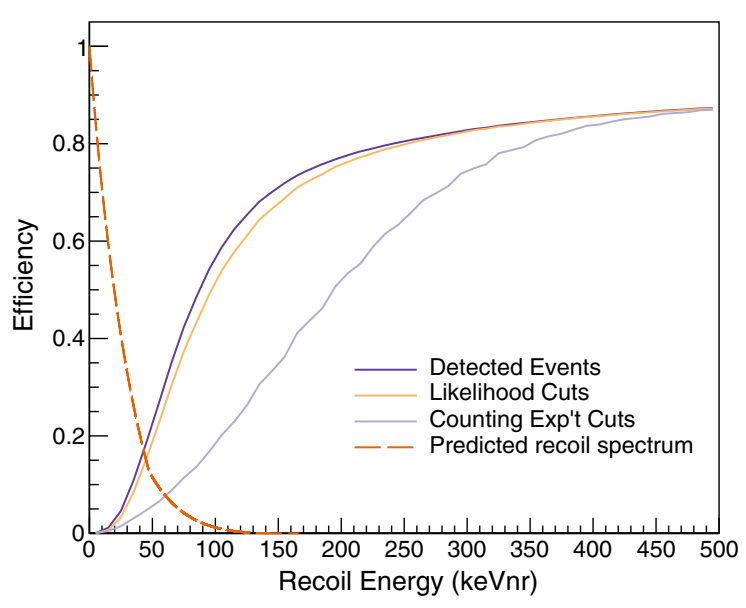

FIG. 5. Estimated efficiency for acceptance of nuclear recoil events in CENNS-10 as function of nuclear recoil energy. "Detected events" are those that pass the $2 \mathrm{PE}$ coincidence required for event building. The likelihood and counting experiment cuts reflect the change in efficiency due to analysis cuts discussed in the text. The overlaid dashed curve is the expected recoil spectrum in argon from CEvNS with arbitrary normalization.

relative to the SNS timing signal, and from $F_{\text {prompt }}$ values ranging from 0.55-0.95. The lack of CEvNS events with reconstructed energy $E_{\text {reco }}>50 \mathrm{keVee}$ and the lack of beam-related neutron events in the delayed window $\left(t_{\text {Trig }}>1.4 \mu \mathrm{s}\right)$ serves to separate the beam-related neutron and CEvNS signals. The efficiencies as a function of nuclear recoil energy for these cuts are seen in Fig. 5.

Systematic errors were assigned to the beam-related (CEvNS and beam-related neutron) predictions for the quenching factor and pulse-finding threshold. These uncertainties were dominated by the uncertainty of the NR PSD band in the CEvNS energy region due to the high threshold of the ${ }^{252} \mathrm{Cf}$ calibration datasets. An additional source of uncertainty was included on the overall beam-related neutron normalization due to the extrapolation of the beam-related neutron rate from the minimal-shielded dataset. For the cuts-based analysis, correlated systematic errors were calculated, and a goodness-of-fit $\left(\chi^{2}\right)$ quantity was determined for the beam excess compared to the MC prediction. For the cross section limits from the likelihood fits, alternative probability density functions incorporating $\pm 1 \sigma$ excursions for each systematic were fit to the data, and the differences from the central value result were added in quadrature as a measure of the systematic uncertainty.

\section{RESULTS}

The resulting sample from the beam-related-neutronenhanced cuts-based analysis (0-700 keVee) over the full time range is shown in Fig. 6. Note the clear evidence of beam-related neutrons with time structure consistent with the POT trace from the SNS beam. Note also that there 


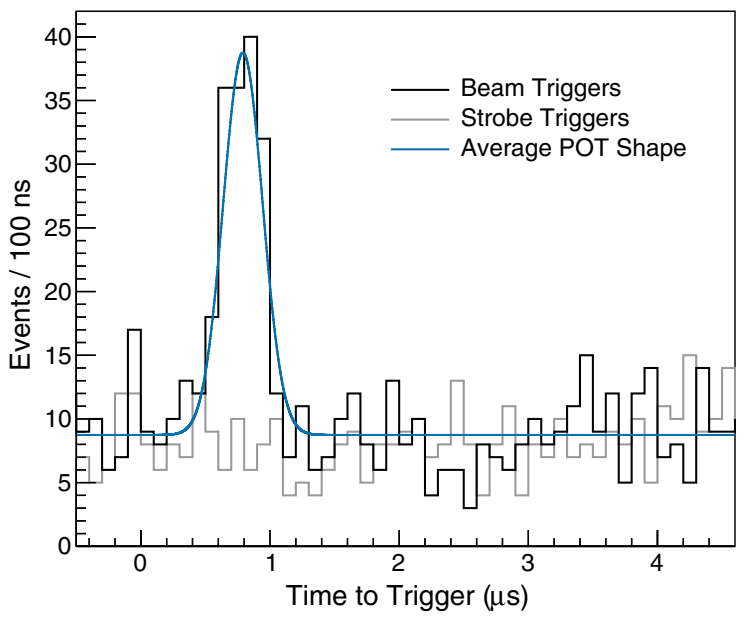

FIG. 6. Time distribution of beam-on and strobe samples in the beam-related-neutron-enhanced energy window. The blue curve is the expectation from the timing shape of the SNS POT signal scaled to the beam-on-target excess.

is no evidence of this signal in the delayed ( $\left.t_{\text {Trig }}>1.4 \mu \mathrm{s}\right)$ region. This is consistent with the hypothesis that the beam-related neutrons that reach the CENNS-10 detector inside of the shielding are the result of fast neutrons in neutrino alley that lose sufficient energy to create lowenergy nuclear recoils in LAr. This was verified by MC simulations.

The reconstructed energy distribution from this sample in the prompt time region $\left(0.4<t_{\text {Trig }}<1.4 \mu \mathrm{s}\right)$ is shown in Fig. 7. The beam-related excess of $126 \pm 15$ (stat.) events in this sample is consistent with the beam-related neutron

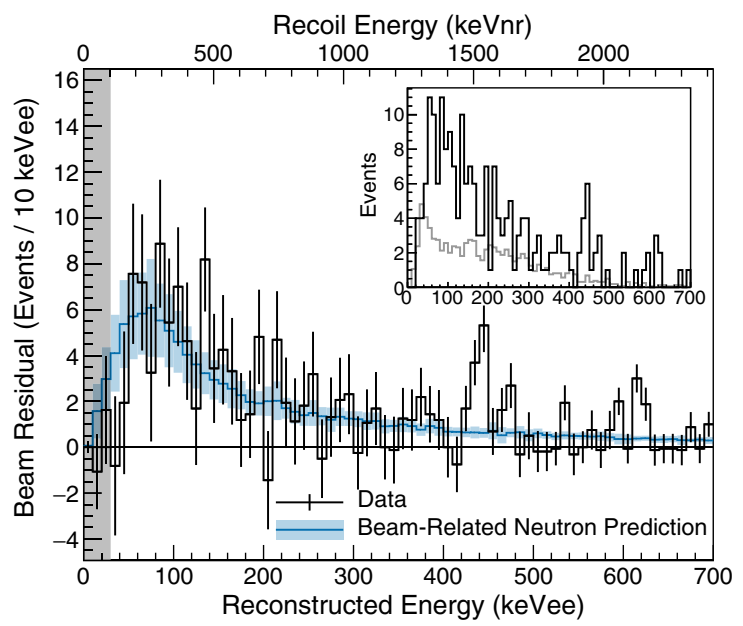

FIG. 7. Energy distribution of the cuts-based analysis beamresidual event sample in the prompt time window along with the beam-related neutron prediction. The error bars are statistical and the error band on the prediction is systematic. Plot inlay shows unsubtracted spectra from the prompt beam-on triggers (black) and the expected beam-unrelated background as measured with strobe triggers (gray). The shaded region corresponds to the CEvNS energy region of interest.

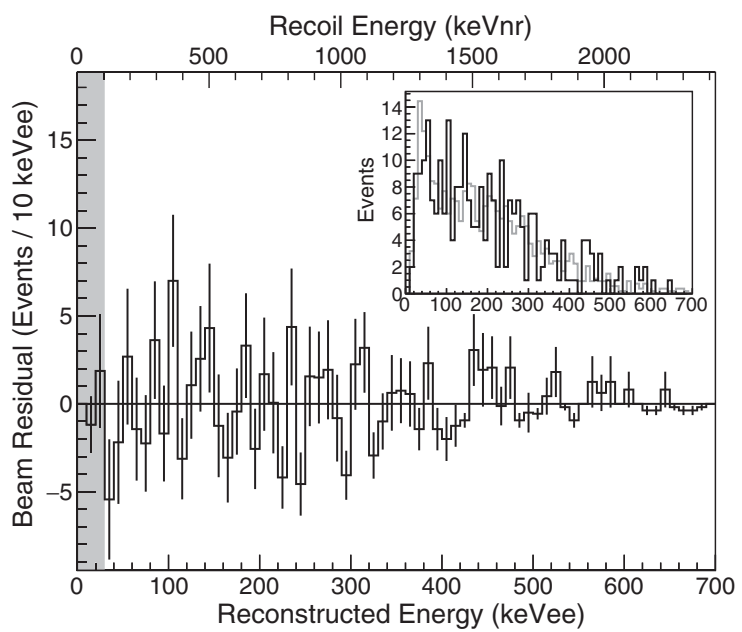

FIG. 8. Energy distribution of the cuts-based analysis beamresidual event sample in the delayed time window. Plot inlay shows unsubtracted beam-on spectrum (black) along with the expected beam-unrelated backgrounds as measured with strobe triggers (gray). The shaded region corresponds to the CEvNS energy region of interest.

prediction of $112 \pm 30$ (syst.) events. The uncertainty on the beam-related neutron prediction is dominated by the uncertainty in the overall fast neutron flux $( \pm 20 \%)$, the uncertainty of the NR PSD band mean near threshold $( \pm 19 \%)$, the pulse-finding threshold $( \pm 5 \%)$, and the quenching factor $( \pm 4 \%)$. The predicted CEvNS signal in this sample is $<1$ detected event. A comparison of the data with the predicted beam-related neutron energy spectrum gives a $\chi^{2} / N_{\text {bins }}$, including correlated uncertainties, of $99 / 70$ (2.0/3 in the CEvNS energy ROI). The origin of the excess at $E \approx 440 \mathrm{keVee}$ is unknown at this time but is believed to be a statistical fluctuation. The excess has a global p-value under the null hypothesis of $1.7 \%$ and is above the energy region of interest for the likelihood fit.

The energy distribution of events in the delayed sample is shown in Fig. 8. In the CEvNS energy region 0-30 keVee, an excess of $1 \pm 4$ (stat.) events is observed, with a predicted CEvNS sample of $<1$ event with an uncertainty dominated by the pulse-finding threshold $( \pm 35 \%)$, the NR PSD band mean behavior near threshold $( \pm 30 \%)$, the quenching factor

TABLE I. Results of a maximum likelihood fit to the data (details in text). The quoted beam-unrelated background counts include the statistical uncertainty in its determination from the strobe trigger sample.

\begin{tabular}{lc}
\hline \hline Sample size & 4663 \\
\hline Beam-unrelated background & $4700 \pm 34$ \\
Fit beam-related neutrons & $126 \pm 18($ stat $) . \pm 28$ (syst) \\
$1 \sigma(68 \%$ CL) CEvNS events & $<7.4$ \\
$1 \sigma$ cross section & $<3.4 \times 10^{-39} \mathrm{~cm}^{2}$ \\
$1 \sigma$ cross section sensitivity & $<7.1 \times 10^{-39} \mathrm{~cm}^{2}$ \\
\hline \hline
\end{tabular}




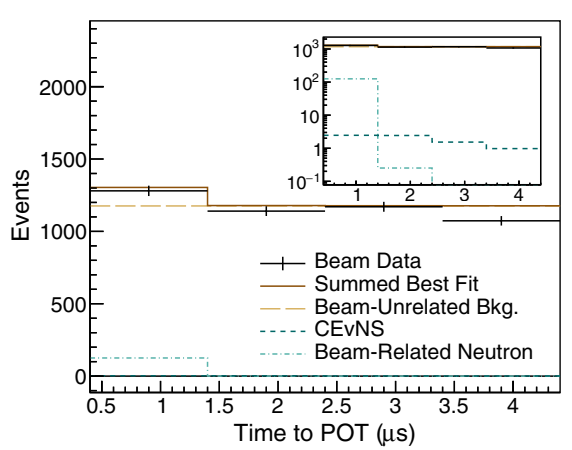

(a)

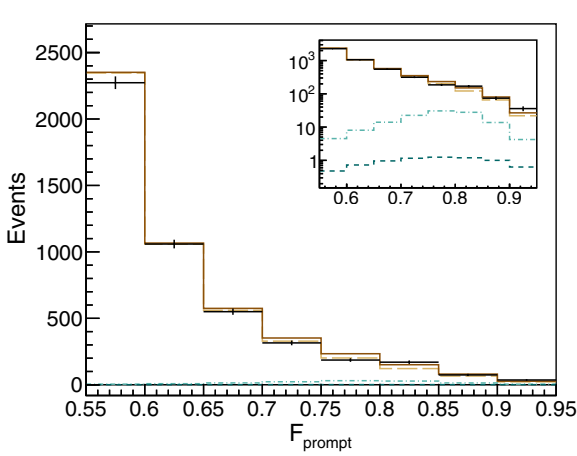

(b)

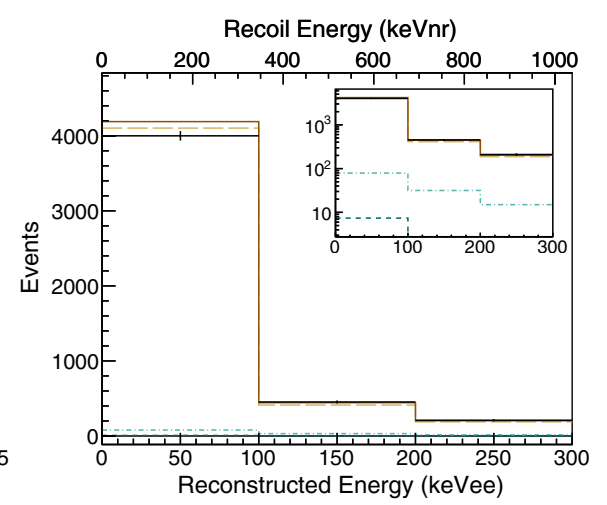

(c)

FIG. 9. Projections of likelihood best-fit solutions together with the data in (a) time, (b) $F_{\text {prompt }}$, and (c) reconstructed energy. The CEvNS curve shown is from the $68 \%$ confidence limit found. Inlaid plots show the spectra in log-scale to make the small contributions from the predicted CEvNS distribution more visible.

$( \pm 15 \%)$, and the uncertainty in the neutrino flux $( \pm 10 \%)$. The first two errors are relatively large because the CEvNS events are so near the threshold in this dataset. In addition, there are $9 \pm 18$ (stat.) events in the extended energy range out to $700 \mathrm{keVee}$, consistent with earlier measurements $[18,44]$ indicating that there is no delayed beam-related neutron flux in neutrino alley.

The likelihood fit was performed by passing a total of 4663 events surviving the likelihood cuts to a 3D likelihood function in energy, time, and $F_{\text {prompt }}$ space including beamunrelated and beam-related neutron backgrounds along with a CEvNS signal. A profile likelihood curve was calculated as a function of the number of CEvNS events and a frequentist confidence limit (CL) method [45-47], along with a simple treatment of the large systematic errors, was used to place on upper limit on the number of CEvNS

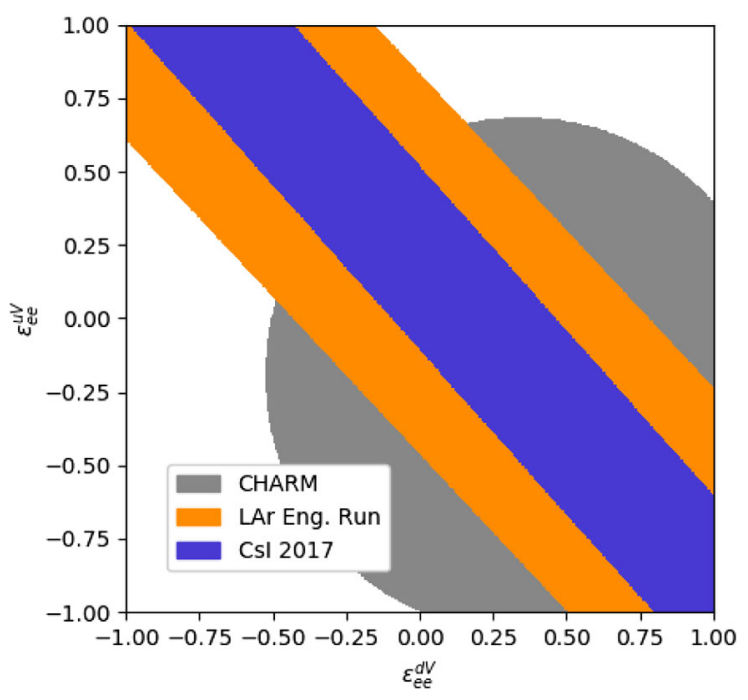

FIG. 10. $90 \% \mathrm{CL}$ on NSI parameters $\epsilon_{e e}^{u V}$ and $\epsilon_{e e}^{d V}$ from this CENNS-10 engineering run. The earlier CsI[Na] result [18] is confirmed and much of the pre-COHERENT phase space allowed by CHARM [49] is ruled out. events of $<7.4$ events. This result can be used to place a $68 \% \mathrm{CL}$ on the stopped-pion flux-averaged cross section of $<3.4 \times 10^{-39} \mathrm{~cm}^{2}$, within twice the SM prediction of $1.8 \times 10^{-39} \mathrm{~cm}^{2}$ [48]. These results are summarized in Table I, and the projections in time, $F_{\text {prompt }}$, and reconstructed energy are shown in Fig. 9.

Using the same frequentist method a $90 \% \mathrm{CL}$ on the cross section of $<8.3 \times 10^{-39} \mathrm{~cm}^{2}$ was extracted and used to set limits on the NSI couplings $\epsilon_{e e}^{u V}, \epsilon_{e e}^{d V}$ [9]. Under the assumption of heavy mediators, these couplings result in an overall scaling factor to the CEvNS cross section [18]. Figure 10 indicates the allowed parameter values consistent with this $90 \%$ CL cross section.

\section{CONCLUSIONS}

In this first result from the CENNS-10 liquid argon detector as part of the COHERENT experiment, a dataset taken as part of an engineering run corresponding to $4.2 \times 10^{22}$ protons on the SNS target collected from February 24, 2017, to May 28, 2017, has been analyzed. The energy threshold in this configuration is not adequate for high sensitivity to CEvNS. However, beam-related neutrons were characterized, further refining constraints on this important background, which will inform future measurements. In addition, no beam-related neutrons were observed in the delayed time window, outside of the beam pulse, consistent with previous measurements. The observation of no significant beam excess allows for a first limit on the CEvNS cross section on argon within twice the SM prediction and for a corresponding limit on NSI.

The CENNS-10 detector was upgraded in summer 2017 to improve light collection and lower the energy threshold to $20 \mathrm{keVnr}$, and additional shielding was installed to minimize the dominant beam-unrelated background in Neutrino Alley. CENNS-10 has collected $>6 \mathrm{GWh}$ of data in this configuration with the sensitivity to make a first 
observation of CEvNS on argon. COHERENT is also working toward CEvNS measurements with a $2 \mathrm{t} \mathrm{NaI}$ detector array, also sensitive to charged current interactions, as well as with $16 \mathrm{~kg}$ p-type point-contact Ge detectors to maximize the neutrino physics capabilities at the SNS [48].

\section{ACKNOWLEDGMENTS}

The COHERENT collaboration acknowledges the generous resources provided by the ORNL Spallation Neutron Source, a DOE Office of Science User Facility, and thanks Fermilab for the continuing loan of the CENNS-10 detector. We also acknowledge support from the Alfred P. Sloan Foundation, the Consortium for Nonproliferation Enabling Capabilities, the Institute for Basic Science (Korea, Grant No. IBS-R017-G1-2019-a00), the National Science Foundation, the Russian Foundation for Basic Research (Project No. 17-02-01077 A), and the U.S.
Department of Energy, Office of Science. Laboratory Directed Research and Development funds from ORNL and Lawrence Livermore National Laboratory also supported this project. This research used the Oak Ridge Leadership Computing Facility, which is a DOE Office of Science User Facility. Notice: This manuscript has been authored by UT-Battelle, LLC, under Contract No. DEAC05-00OR22725 with the U.S. Department of Energy (DOE). The U.S. government retains and the publisher, by accepting the article for publication, acknowledges that the U.S. government retains a nonexclusive, paid-up, irrevocable, worldwide license to publish or reproduce the published form of this manuscript, or allow others to do so, for U.S. government purposes. DOE will provide public access to these results of federally sponsored research in accordance with the DOE Public Access Plan (http://energy .gov/downloads/doe-public-access-plan).
[1] D. Z. Freedman, Phys. Rev. D 9, 1389 (1974).

[2] V. B. Kopeliovich and L. L. Frankfurt, Pis'ma Zh. Eksp. Teor. Fiz. 19, 236 (1974) [JETP Lett. 19, 145 (1974)].

[3] P. S. Amanik and G. C. McLaughlin, J. Phys. G 36, 015105 (2009).

[4] M. Cadeddu, C. Giunti, Y. F. Li, and Y. Y. Zhang, Phys. Rev. Lett. 120, 072501 (2018).

[5] M. Cadeddu and F. Dordei, Phys. Rev. D 99, 033010 (2019).

[6] K. Patton, J. Engel, G. C. McLaughlin, and N. Schunck, Phys. Rev. C 86, 024612 (2012).

[7] D. A. Sierra, J. Liao, and D. Marfatia, J. High Energy Phys. 06 (2019) 141.

[8] M. Hoferichter, P. Klos, J. Menéndez, and A. Schwenk, Phys. Rev. D 99, 055031 (2019).

[9] J. Barranco, O. G. Miranda, and T. I. Rashba, J. High Energy Phys. 12 (2005) 021.

[10] K. Scholberg, Phys. Rev. D 73, 033005 (2006).

[11] J. Barranco, O. G. Miranda, and T. I. Rashba, Phys. Rev. D 76, 073008 (2007).

[12] B. Dutta, R. Mahapatra, L. E. Strigari, and J. W. Walker, Phys. Rev. D 93, 013015 (2016).

[13] D. K. Papoulias and T. S. Kosmas, Phys. Rev. D 97, 033003 (2018).

[14] L. M. Krauss, Phys. Lett. B 269, 407 (1991).

[15] P. Coloma and T. Schwetz, Phys. Rev. D 94, 055005 (2016); 95, 079903(E) (2017).

[16] P. Coloma, P. B. Denton, M. C. Gonzalez-Garcia, M. Maltoni, and T. Schwetz, J. High Energy Phys. 04 (2017) 116.

[17] P. Coloma, M. C. Gonzalez-Garcia, M. Maltoni, and T. Schwetz, Phys. Rev. D 96, 115007 (2017).

[18] D. Akimov et al. (COHERENT Collaboration), Science 357, 1123 (2017).

[19] R. Tayloe (COHERENT Collaboration), J. Instrum. 13, C04005 (2018).

[20] S. J. Brice et al., Phys. Rev. D 89, 072004 (2014).
[21] V. M. Gehman, S. R. Seibert, K. Rielage, A. Hime, Y. Sun, D. M. Mei, J. Maassen, and D. Moore, Nucl. Instrum. Methods Phys. Res., Sect. A 654, 116 (2011).

[22] C. Benson, G. Orebi Gann, and V. Gehman, Eur. Phys. J. C 78, 329 (2018).

[23] D. N. McKinsey, C. R. Brome, J. S. Butterworth, R. Golub, K. Habicht, P. R. Huffman, S. K. Lamoreaux, C. E. H. Mattoni, and J. M. Doyle, Nucl. Instrum. Methods Phys. Res., Sect. B 132, 351 (1997).

[24] P. Agnes et al. (DarkSide Collaboration), Phys. Lett. B 743, 456 (2015).

[25] P. Agnes et al. (DarkSide Collaboration), Phys. Rev. D 98, 102006 (2018).

[26] R. Ajaj et al. (DEAP Collaboration), Phys. Rev. D 100, 022004 (2019).

[27] C. Adams et al. (The MicroBooNE Collaboration), Phys. Rev. D 99, 091102 (2019).

[28] T. Doke, K. Masuda, and E. Shibamura, Nucl. Instrum. Methods Phys. Res., Sect. A 291, 617 (1990).

[29] D. Gastler, E. Kearns, A. Hime, L. C. Stonehill, S. Seibert, J. Klein, W. H. Lippincott, D. N. McKinsey, and J. A. Nikkel, Phys. Rev. C 85, 065811 (2012).

[30] H. Cao et al. (SCENE Collaboration), Phys. Rev. D 91, 092007 (2015).

[31] W. Creus, Y. Allkofer, C. Amsler, A. D. Ferella, J. Rochet, L. Scotto-Lavina, and M. Walter, J. Instrum. 10, P08002 (2015).

[32] P. Agnes et al., Phys. Rev. D 97, 112005 (2018).

[33] A. Hitachi, T. Takahashi, N. Funayama, K. Masuda, J. Kikuchi, and T. Doke, Phys. Rev. B 27, 5279 (1983).

[34] P. A. Amaudruz et al. (DEAP Collaboration), Astropart. Phys. 85, 1 (2016).

[35] P. Benetti, R. Acciarri, F. Adamo, B. Baibussinov, M. Baldo-Ceolin, M. Belluco, F. Calaprice, E. Calligarich, M. Cambiaghi, and F. Carbonara, Astropart. Phys. 28, 495 (2008). 
[36] W. H. Lippincott, K. J. Coakley, D. Gastler, A. Hime, E. Kearns, D. N. McKinsey, J. A. Nikkel, and L. C. Stonehill, Phys. Rev. C 78, 035801 (2008); 81, 039901(E) (2010).

[37] H. O. Back, A. Alton, F. Calaprice, C. Galbiati, A. Goretti, C. Kendziora, B. Loer, D. Montanari, P. Mosteiro, and S. Pordes, Phys. Procedia 37, 1105 (2012).

[38] H. O. Back et al., arXiv:1204.6024.

[39] P. Agnes et al. (DarkSide Collaboration), Phys. Rev. D 93, 081101 (2016); 95, 069901(A) (2017).

[40] R. Cooper, L. Garrison, H. O. Meyer, T. Mikev, L. Rebenitsch, and R. Tayloe, in Particles and Fields. Proceedings, Meeting of the Division of the American Physical Society, DPF 2011, Providence, USA, 2011 (2011).

[41] M. R. Heath, A first search for coherent elastic neutrinonucleus scattering with liquid argon, Ph.D. thesis, Indiana U., Bloomington (main), 2019, https://scholarworks.iu.edu/ dspace/handle/2022/23188.
[42] R. Acciarri et al. (WArP Collaboration), J. Instrum. 5, P06003 (2010).

[43] S. Agostinelli et al. (GEANT4 Collaboration), Nucl. Instrum. Methods Phys. Res., Sect. A 506, 250 (2003).

[44] D. Akimov et al. (COHERENT Collaboration), arXiv:1509 .08702 .

[45] G. J. Feldman and R. D. Cousins, Phys. Rev. D 57, 3873 (1998).

[46] R. T. Thornton, Search for light dark matter produced in a proton beam dump, Ph.D. thesis, Indiana U., 2017, https:// www.osti.gov/biblio/1345594/.

[47] A. A. Aguilar-Arevalo et al. (MiniBooNE Collaboration), Phys. Rev. Lett. 118, 221803 (2017).

[48] D. Akimov et al. (COHERENT Collaboration), arXiv:1803 .09183 .

[49] J. Dorenbosch et al. (CHARM Collaboration), Phys. Lett. B 180, 303 (1986). 